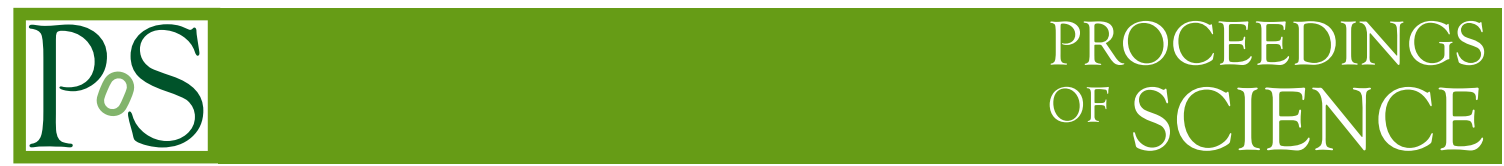

\title{
CMS detector commissioning results with cosmics
}

\author{
Mario Pelliccioni* \\ Università degli Studi di Torino, Istituto Nazionale di Fisica Nucleare \\ E-mail: pellicci@cern.ch
}

We review the status of the commissioning of the Compact Muon Solenoid experiment, with a focus on the study of the detector performance determined using cosmic muons. The results presented here are obtained using the 2008 cosmics data.

12th International Conference on B-Physics at Hadron Machines - BEAUTY 2009 September 07 - 112009

Heidelberg, Germany

\footnotetext{
* Speaker.
} 


\section{Introduction to the CMS detector}

Operating on the LHC machine at CERN, the Compact Muon Solenoid (CMS) is an experiment designed to achieve an excellent particle identification and therefore profit of the rich physics program that the $14 \mathrm{TeV}$ proton collider can deliver. A detailed description of the CMS goals is reported in [1]. CMS [2] relies on a super-conducting solenoid to provide a $4 T$ magnetic field. Inside the field volume, the tracker and the calorimeters are found. The muon spectrometer is located outside the field, in the iron yoke. A schematic picture of the experiment is shown in Fig. 1

The purpose of the CMS tracker is to characterize the trajectories of charged particles from LHC with a high level of precision and efficiency. It must meet the resolution requirements needed in order to discriminate secondary vertexes. The inner part of the tracker is composed by pixel detectors, which consist of three cylindrical layers in the barrel and two disks in each endcap, for a total of 66 million channels. The outer part is composed by strip detectors. In the barrel, it is divided in two concentric subdetectors of increasing radius of four and six layers (TIB and TOB respectively). In the endcaps, it is divided in two disks per side, each of three and nine layers (TIF and TEC respectively). The total number of channels for the strips tracker is 11 million.

CMS is equipped with electromagnetic and hadronic calorimeters. The electromagnetic calorimeter (ECAL) is composed by 76000 lead tungstate $\left(\mathrm{PbWO}_{4}\right)$ crystals. It is designed to have an energy resolution below $0.5 \%$ above the energy of $100 \mathrm{GeV}$. The hadronic calorimeter (HCAL) is made of 70000 brass scintillator plates. An additional hadron detector is located outside the barrel region occupied by HCAL, and provides additional information to reject residual hadronic showers. These calorimeters cover an acceptance of $|\eta|<3$, where $\eta$ is the pseudorapidity. The acceptance region of $3<\eta<5$ is covered by a quartz very forward calorimeter. The combined ECAL and HCAL resolution on measured jets is $\Delta E / E=100 \% / \sqrt{(} E) \oplus 5 \%$.

In the barrel yoke, one can find the muon spectrometer. This is a very important detector for CMS in terms of the ability to perform physics analysis at the LHC. Given the high amount of background expected in a proton-proton machine, final states containing muons often provide a clean signature. The barrel is divided into five wheels, and is equipped with Drift Tube (DT) chambers and Resistive Place Chambers (RPC) with large redundancy. The RPCs provide a precise measurement of the timing, that allows bunch crossing determination, and an additional measurement of the particle momentum. Each wheel is segmented into twelve sectors and covers the full azimuthal angle. Each sector consists of four layers of DT chambers and six layers of RPCs. Apart from the outermost layer, each DT layer is made of three groups of four layers of tubes, also called Super Layers (SL). Each SL can reconstruct the three dimensional trajectory, or segment, using up to eight hits in the $\mathrm{r}-\phi$ plane and up to four hits in the r-z plane. The outermost DT layer can only provide a segment in the $r-\phi$ view. The endcaps (four independent disks for each side) are equipped with Cathode Strip Chamber (CSC) detectors and RPC. The muon system covers a pseudorapidity region of $|\eta|<2.4$. The reconstruction is able to match the muons detected in the spectrometer with the tracks in the silicon tracker, for a transverse momentum resolution lower than $5 \%$ for $p_{T}<1$ $\mathrm{TeV} / \mathrm{c}$. 


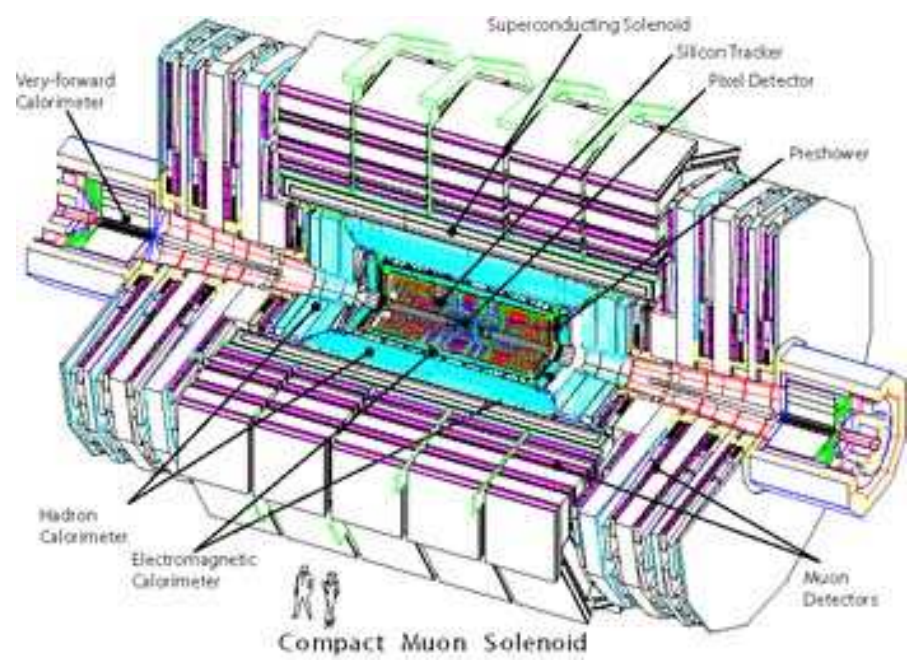

Figure 1: The CMS detector.

\section{The CMS commissioning}

CMS was designed so that the detector construction could be performed on surface. After being cabled and partially commissioned, the subsystems were lowered in the pit where the experiment now resides. The assembly took place between 2001 and 2006, and ended with the close of the detector and the commissioning phase of the system as a whole. In 2006, as a preliminary test, a portion of all subsystems was used to detect cosmic rays, to determine the ability of the experiment to combine the information from the single subdetectors. Moreover, a preliminary mapping of the magnetic field was performed. This test was called the Magnet Test and Cosmic Challenge (MTCC) [3].

In 2007, 2008 and 2009 CMS undertook several campaigns, often referred to as global runs to collect cosmic data and test the detector. These data takings where performed both without the magnetic field (and were called Cosmic RUn at Zero Tesla, CRUZET) and with the magnetic field at its nominal value (called Cosmic Run At Four Tesla, CRAFT).

\subsection{The 2008 CRAFT}

In the period between October and November 2008, CMS undertook a CRAFT data taking that collected about 300 million cosmic muon events. About 200 million of these events were taken with the whole detector running. This huge amount of data was crucial to study the detector performance. It also proved that the single subsystems were able to collect data on a longer period of time. Calibration and alignment constants obtained studying this data allowed a better understanding of the detector. Furthermore, the Data Quality Monitor (DQM) and the Detector Control System (DCS) where used through the data taking. This exercise allowed to test and refine them. Figure 2 reports the number of collected events as a function of time. 


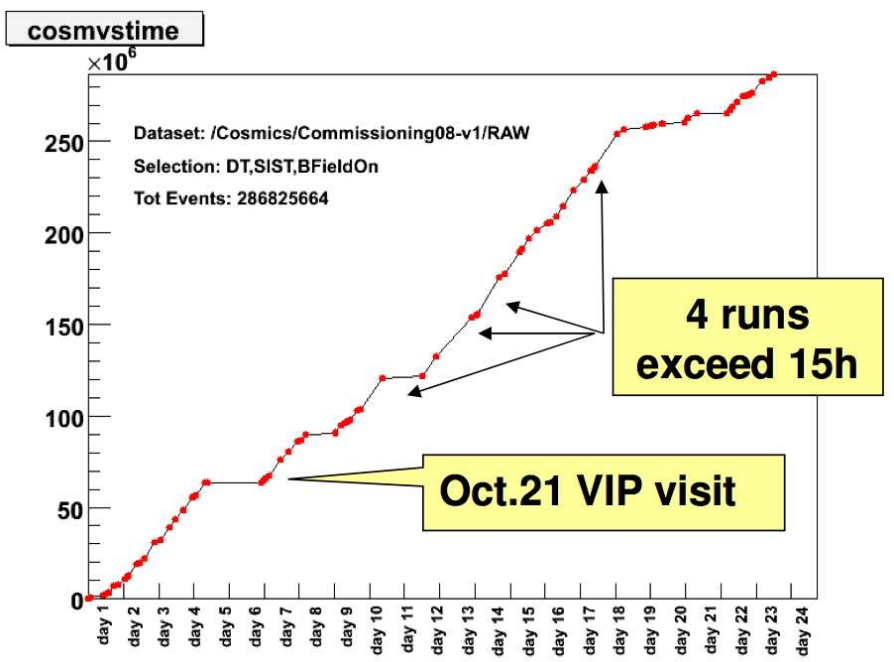

Figure 2: Number of collected events during CRAFT 2008 as a function of time.

\section{Study of CMS performance with cosmic data}

In the following, we report the results of the CMS studies obtained using cosmic data. Unless otherwise stated, these results refer to the 2008 CRAFT.

\subsection{The silicon tracker}

The signal-to-noise ratio was characterized using the cosmic data. This ratio was determined separately for each layer, as it depends on the thickness of the module. The estimation was done selecting clusters associated with a track only. The incident angle of the cosmic muon was considered in the correction. The values of the ratio are all between 25 and 30 . The efficiency of the algorithm that associates the hits to a track was measured to be higher than $99 \%$. Some regions of the tracker presented lower efficiencies, but those corresponded to known problems.

Alignment was performed using events with reconstructed tracks in the tracker. In Fig. 3 the distribution of the median of the residual for several strip components is shown. All the residuals are compatible with zero within the error.

Lastly, the drift motion of the charge carriers was studied. The Lorentz force due to the presence of the magnetic field influences the drift velocity. To determine the angle at which the Lorentz force deflects the velocity, we measured the spread of the drift charge distribution as a function of the incident angle of the track, and determined the minimum. For the barrel pixel, the value of this angle is $24.60^{\circ}$.

\subsection{The calorimeters}

The deposited energy of muons divided by the path length in the crystal or the tower (stopping power) was measured as a function of momentum for both ECAL and HCAL. In Fig. 4a the ECAL theoretical distribution (full line) for the stopping power in $\mathrm{PbWO}_{4}$ is compared with the 

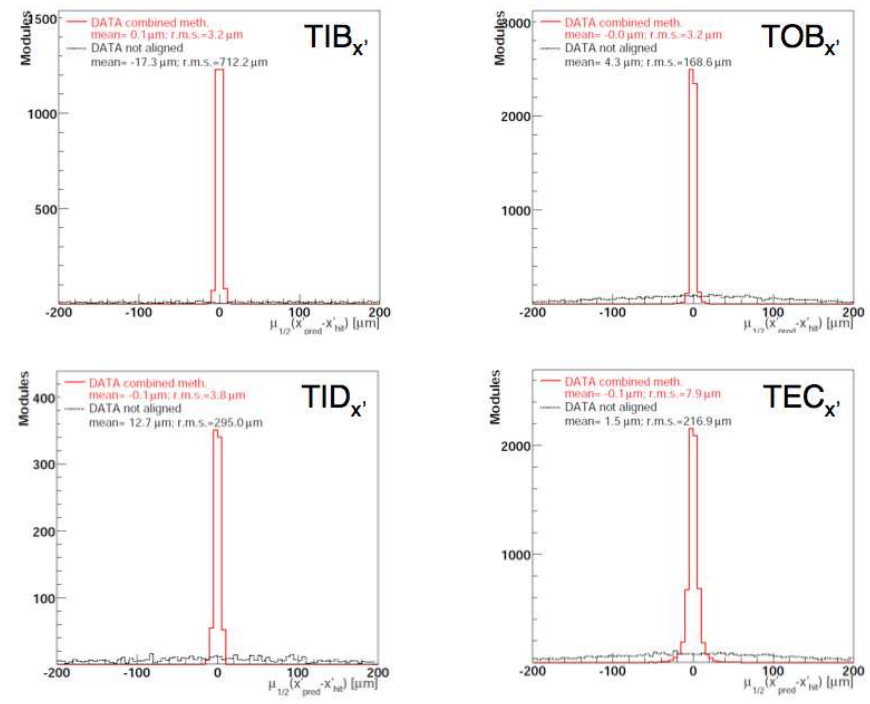

Figure 3: Median of the residuals for several strips components, determined using the CRAFT 2008 data.
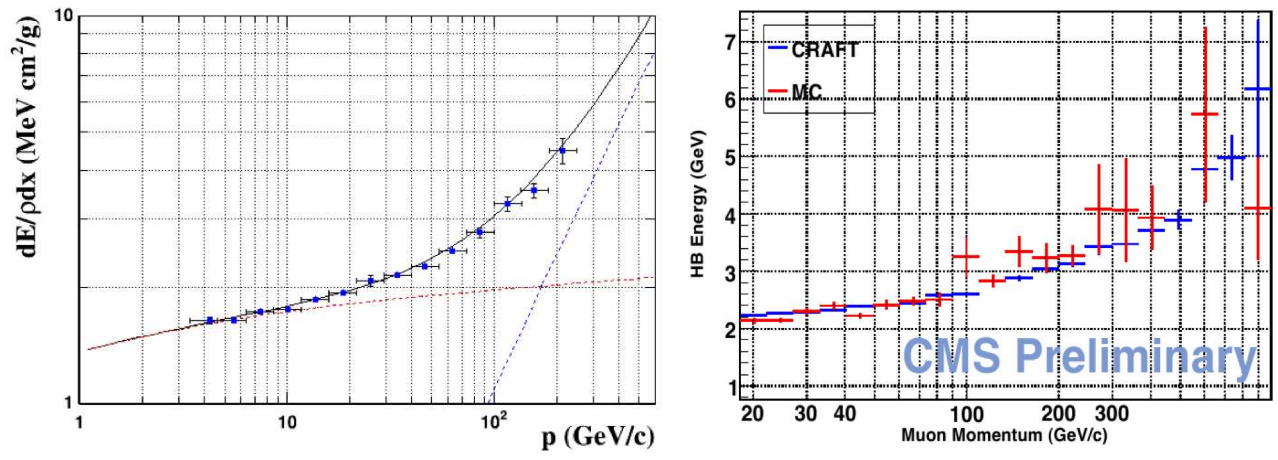

Figure 4: (a) Theoretical distribution (black line) and measurement of the stopping power of ECAL crystals as a function of momentum. The red dashed line corresponds to the theoretical prediction for collision losses, while the blue one is the contribution for bremsstrahlung. (b) Theoretical prediction (red) and measured (blue) energy loss in HCAL as a function of momentum.

experimental data. In Fig. $4 \mathrm{~b}$ the same exercise is done for HCAL. In both cases a good agreement is found between data and theoretical predictions, indicating that both the tracker momentum determination and the calorimeter energy scales are correct.

\subsection{The muon spectrometer}

The commissioning of the muon detector is naturally the one that profited the most of the cosmic data taking. We studied and improved the reconstruction algorithms and the muon identification software.

As far as the DT are concerned, we tested the efficiency of the local reconstruction at the level of the single cell. This is done by comparing the measured hit position in the cell with the expected 

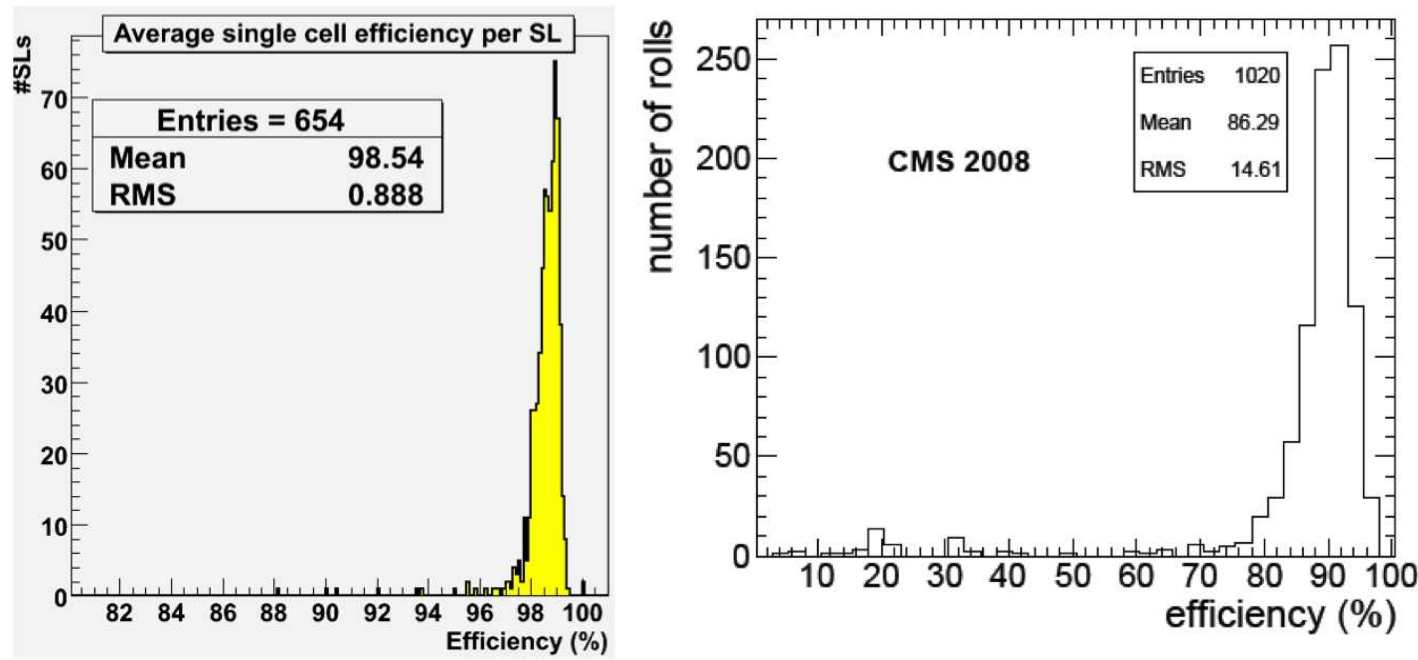

Figure 5: (a) Average single cell efficiency for the DT Super Layers in CRAFT 2008. (b) Maximum efficiency for all the RPCs in the barrel system in CRAFT 2008.

position of the fitted track. In Fig. 5a we report the distribution of the average cell efficiency of the Super Layers. The left, limited, tail in the efficiency distribution is due to known problems that were fixed during the following shutdowns. We also calculated the resolution on the position of the hits, and it was found to be between 200 and $260 \mu \mathrm{m}$, in good agreement with the simulation predictions. Some regions were found where the residual magnetic field distorts the drift velocity inside the cell. This phenomenon was found in the innermost stations of the most external wheels of the barrel. The resolution in those chambers is worse with respect to the average values.

We also studied the RPCs efficiency by extrapolating in the chambers the muon trajectory reconstructed in the DT system and looking for hits in the RPC in a region of \pm 2 strips. This study was done at the strip level to better determine inefficient areas and to characterize the uniformity of the detector. The voltage at which the chambers operate was varied and the efficiencies were measured, to better determine the best voltage for data taking. In Fig. $5 \mathrm{~b}$ we present the maximum efficiency of the chamber for all the RPCs. Again, the left tails were due to known hardware problems, most of which were fixed in the subsequent shutdown.

\section{Conclusions}

CMS undertook several campaigns that allowed the collection of large data samples of cosmic muons. With this data, it was possible to evaluate the performance of the experiment as a whole and of its subdetectors. Calibrations and alignement of the system allowed to reach the expected resolution and efficiencies. The detector is ready for continuous data taking during the LHC operation. 


\section{References}

[1] G. L. Bayatian et al. [CMS Collaboration], "CMS physics: Technical design report," CMS CERN/LHCC 2006-001

[2] R. Adolphi et al. [CMS Collaboration], "The CMS experiment at the CERN LHC," JINST 0803, S08004 (2008) [JINST 3, S08004 (2008)].

[3] CMS Collaboration, “The CMS Magnet Test and Cosmic Challenge (MTCC Phase I and II)," CMS Note/2007-005, CERN/LHCC 2007-011. 